\title{
Simulation of small size divertor tokamak plasma edge at low density of plasma
}

\author{
Amr Hasheim Bekheit \\ Plasma and Nuclear Fusion Department, Atomic Energy Authority, Cairo, Egypt; amrbekheitga@yahoo.com
}

Received 21 August 2011; revised 30 September 2011; accepted 6 October 2011

\begin{abstract}
A low density plasma edge of small size divertor tokamak has been modeling by "B2SOLPS0.5.2 D" fluid transport code. The results of modeling are: 1) Formation of the strong "ITB" has detected more reliable with discovery that, low density plasma is necessary and important condition for it to form; 2) Reduction of plasma density play significantly role in the formation of the strong ITB as global parameter, possibly through change in the steep density gradient which stabilize "ITG" mode; 3 ) The radial electric field of small size divertor tokamak plasma edge is plasma density dependence and maximum radial electric field shear is found at low plasma density; 4) In the "NBI" discharge the toroidal (parallel) velocity at low plasma density is cocurrent and upward direction; 5) The structure of plasma pressure and radial electric field in quiescent $\mathrm{H}$-mode are obtained.
\end{abstract}

Keywords: Low Density; Quiescent H-Mode; B2SOLPS Code

\section{INTRODUCTION}

The internal transport barrier "ITB" is a region characteristic by higher confinement in plasma core. The transport coefficients are strongly reduced in this region and steep density and temperature gradients are formed near last close flux surface (separatrix). The internal transport barrier "ITB" regime is an advanced operation scenario presently studied on the various tokamaks (e.g. JET [1], ASDEX Upgrade [2]...) with a view to its extension to next step, large fusion devices A great deal of progress has been made in recent years in theoretical modeling [1, 3-6] and experiment control [7-9] of "ITB". However, the underlying mechanisms for "ITB" formation and sustainment are not yet fully understood [6]. Turbulence suppression in the region of "ITB" is thought to be controlled by interaction of different physical mechanisms of which there are several commonly studied aspects. These include $\mathrm{E} \times B$ flow that stabilizes ion or electron temperature gradient mode or electron modes [10-12]. Improved confinement due to the formation of an internal transport barrier (ITB) has been studied in order to develop an advanced tokamak operation scenario [13,14]. It important to clarify what condition is required for formation of ITBs. Since many parameters appear to affect the conditions for ITB formation (e.g. applying sufficient heating power, toroidal momentum input...). Since the reduced of plasma density for ITB formation is one of the critical issues for the application of ITBs to many tokamaks, it is important to investigate the relation between ITB formation and reduced plasma density. ITB's on small size divertor tokamak are produced by applying neutral beam injection NBI [15]. The detailed study into the reduction of plasma density condition for ITB formation has thus far not been undertaken at small size divertor tokamak. In order to understanding of "ITB" formation at low density plasma, discharge with neutral beam injection "NBI" has been modeling by B2SOLPS0.5.2D fluid transport code [16,17] and analyses are presented in this paper for edge plasma of small size divertor tokamak. The results of modeling demonstrated that, "ITBs" at edge plasma of small size divertor tokamak strong form at low plasma density, due to a steeper normalized density gradient.

\section{THE MAIN RESULTS OF SIMULATION}

The case of unbalance neutral beam injection for the for parameters of Small Size Divertor Tokamak $(\boldsymbol{R}=\mathbf{0 . 3}$ $\mathrm{m}, \boldsymbol{a}=\mathbf{0 . 1} \mathrm{m}, I=50 \mathrm{KA}, B_{T}=1.7 \mathrm{~T}$ ) was chosen for simulation by B2SOLPS0.5.2D fluid transport with average density $\left(4 \leq \boldsymbol{n}_{\boldsymbol{i}} \leq 1\right) \times 10^{19} \mathrm{~m}^{-3}$ and temperature heating $\boldsymbol{T}_{\boldsymbol{i}}=3.443 \mathrm{KeV}$. The simulations were performed with B2SOLPS0.5.2D fluid transport code. As in similar codes the set of modified Braginski equations was solved $[16,17]$. The philosophy B2SOLPS0.5.2D fluid transport code (and other codes) is that the values of perpendicular transport coefficients are chosen to fit experimentally observed density, temperature radial profiles, density and temperature near the divertor plates. In the simulation 
presented below the perpendicular transport coefficients are replaced by the anomalous values: diffusion, electron, ion heat flux and perpendicular viscosity coefficients $[16,17]$. The perpendicular (anomalous) viscosity coefficient was taken in the form $\boldsymbol{\eta}=\mathbf{n} \cdot \mathbf{m}_{\mathbf{i}} \cdot \mathbf{D}$. At the inner boundary flux surface, which was located few $\mathrm{cm}$ from the separatrix, the density, the electron and ion heat fluxes and the average toroidal momentum flux were specified $[16,17]$. The boundary heat fluxes were imposed independently from the toroidal momentum flux thus providing the opportunity to investigate the dependence of radial electric field on these parameters $[16,17]$. The anomalous values of the diffusion electron and ion heat conductivity coefficients were chosen equal for all sort of particle $\boldsymbol{D}=$ $0.5 \mathrm{~m}^{2} \cdot \mathrm{s}^{-1}, \chi_{e, i}=0.7 \mathrm{~m}^{2} \cdot \mathrm{s}^{-1}$. The main results of simulation are:

1) The first result of simulated shows that, the typical profile of plasma density at outer mid-plane is shown Figures 1 and 2. From Figure 2, it can be seen the strong ITB discharge form at density lower than the non-ITB discharge. It seems that, for non-ITB discharges when the condition would otherwise facilitate ITB formation, the plasma density is too high and thus TTB does not form. This hypothesis is supported by ITB discharges, in single null small size divertor tokamak edge plasma, where heating during the current ramp was delayed some time. This delayed heating prevents the neutral beam fuelling from generating higher density before, formation of ITB. This result agrees with the result given by [18].

2) The radial profile of the plasma density characteristic scale length $\left(L_{n}=\left|\partial \ln n / h_{y} \partial y\right|^{-1}, h_{y}\right.$ is one of the metric coefficient) is shown in Figure 3. Figures 1 and 2 show that, by lowering the plasma density, the strong ITB, steep plasma density characteristic scale length and steep plasma density are formed. According to [18-20] the steep plasma density is the main factor for stabilized the turbulence driven by ITG modes.

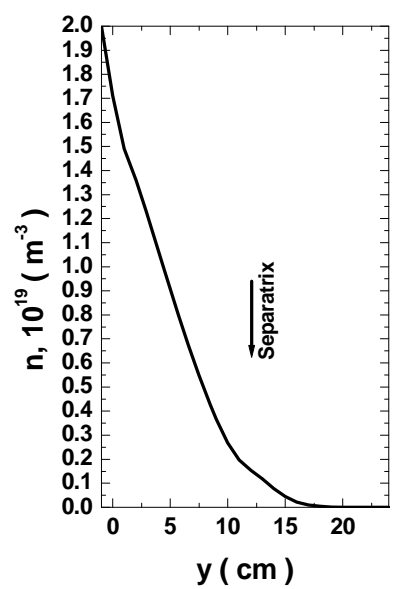

Figure 1. The radial profile of plasma density at lower plasma density in outer mid-plane.

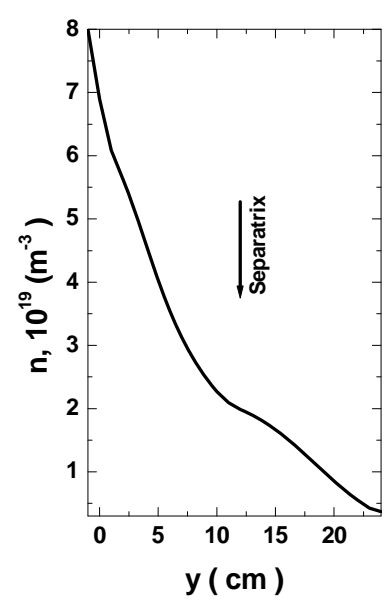

Figure 2. The radial profile of plasma density at lower plasma density in outer mid-plane.

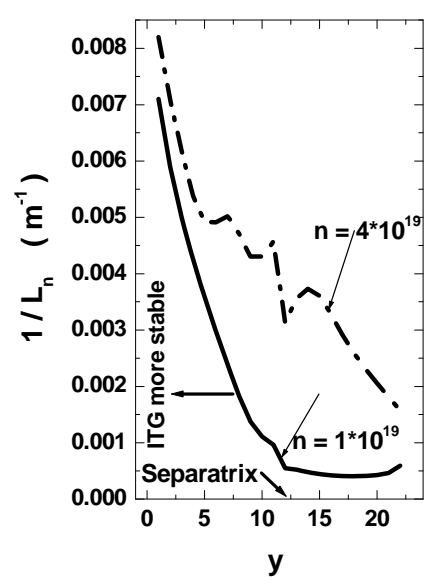

Figure 3. The radial distribution of density gradient length in edge of SSDT at different plasma densities.

A possible explanation for the necessary condition of lower plasma density for steep plasma density and ITB formation is that, by slightly lowering the plasma density and keeping the temperature plasma heating and toroidal torque the same, the radial electric field shear and toroidal velocity will increase, possibly allowing the turbulence to be suppressed. Therefore, we conclude reduction of plasma density play significantly role as global parameter in the formation of strong ITB and steep density gradient which stabilize "ITG” mode.

3) The radial electric field profile for edge plasma of small size divertor tokamak at outer mid-plane is shown in Figure 4. Form Figure 4, it can be seen that lower plasma density has strong influence on the radial distribution of radial electric field. One can see strong spikes form inside separatrix at large reduction of plasma density. The existence of the spike is very important for $\mathrm{L}-\mathrm{H}$ transition and turbulent suppression in single null small 
size divertor tokamak plasma edge. The amplitude of this spike is dependent on plasma density and change in torodial flow flux in the SOL which transport through the separatrix due to anomalous viscosity [21] (as shown in Figure 4). The second spike in the radial electric field too far from separatrix is also important for turbulence suppression and ITB formation. This result is interesting since they might help explain the easier transition to $\mathrm{H}$-regime and strong ITB formation in edge plasma of small size divertor tokamak.

4) The parallel (toroidal) velocity at the outer mid-plane is shown in Figure 5. From Figure $\mathbf{5}$ it can be seen that at low plasma density the toroidal velocity is completely negative in edge plasma of small size divertor tokamak which corresponding to co-current (upward) direction. Figure 5 also shows that, the absolute value of toroidal rotation is larger for lower edge plasma density.

5) The parallel (toroidal) velocity at the outer midplane is shown in Figure 5. From Figure 5 it can be seen that at low plasma density the toroidal velocity is completely negative in edge plasma of small size divertor tokamak which corresponding to co-current (upward) direction. Figure 5 also shows that, the absolute value of toroidal rotation is larger for lower edge plasma density. The reason for the deviation of toroidal velocity at low plasma density from the toroidal velocity at high plasma density can be explaining according [21] as follow: the difference in toroidal velocities at different plasma densities refer to unbalance toroidal (parallel) particle flux caused by radial flux from the plasma core goes to SOL and divertor plates. The strong co-current parallel (toroidal) flux at mid-plane edge plasma of small size divertor tokamak corresponds to co-current Pfirsch-Schlueter flux [22] observed in this tokamak. This result is interesting since they might help explain, The shear of toroidal velocity at low plasma density near separatrix is smaller in case of the high plasma density which explains the larger L-H transition than for high plasma density.

6) The radial profile for low plasma density QH (quiescent $\mathrm{H}$ ) regime plasma pressure as shown in Figure 6. The QH-mode edge radial electric field at low plasma density is shown in Figure 4. The width of the radial electric field inside separatrix is about $\sim 1 \mathrm{~cm}$ close to gyro-radius of ion in the toroidal magnetic field at radius well $\sim 0.9 \mathrm{~cm}$. The structure of the radial electric field at QH shows the value of radial electric field at spike is about $100 \mathrm{k} \mathrm{v} / \mathrm{m}$ compared with $20-40 \mathrm{k} \mathrm{v} / \mathrm{m}$ in H-regime. which consistent with results [23]. This result indicates, the steady stat nature of QH-regime allow us to obtain high enough radial electric field shear for turbulence suppression and strong ITB formation. The simulation of small size divertor tokamak plasma edge in QH-regime by using the B2SOLPS0.5.2D fluid transport code is the subject of the future work.

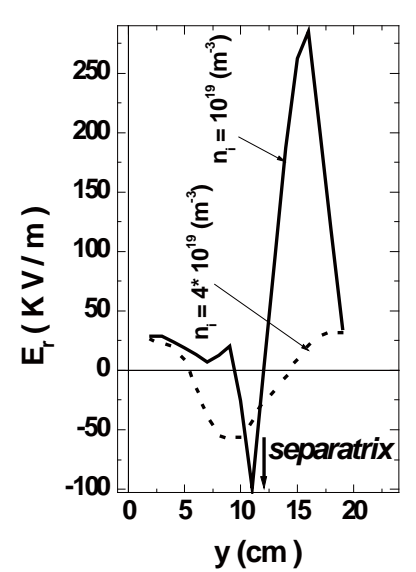

Figure 4. The radial electric field distribution in edge plasma of SSDT at different plasma densities.

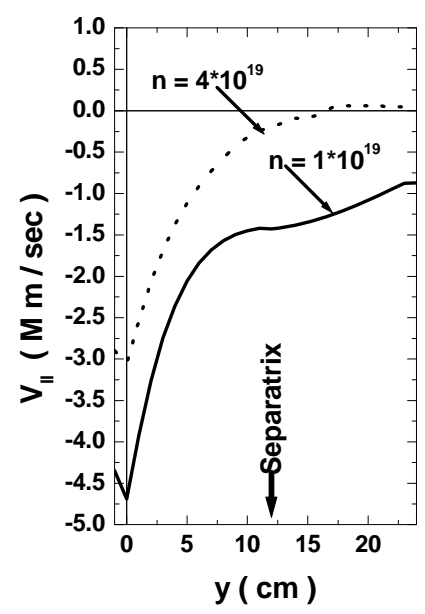

Figure 5. The radial distribution of toroidal velocity at different plasma density.

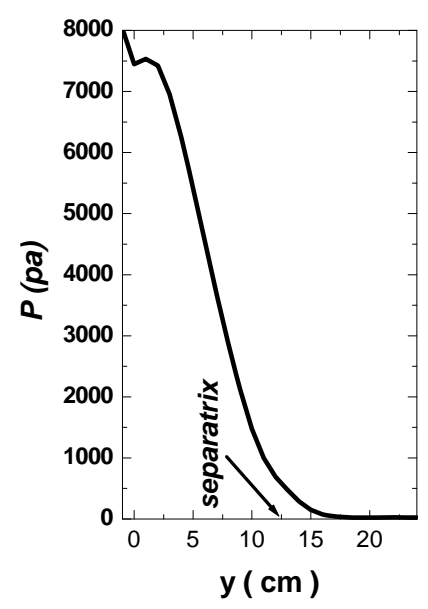

Figure 6. The radial distribution of plasma pressure at QH-regime in edge of SSDT. 


\section{CONCLUSIONS}

The main simulation results demonstrated the following:

1) Formation of the strong "ITB" has detected more reliable with discovery that, low density plasma is necessary and important condition for it to form.

2) Reduction of plasma density play significantly role in the formation of the strong ITB as global parameter, possibly through change in the steep plasma density gradient which stabilize "ITG" mode.

3) The impact of change parallel (toroidal) rotation which transport from SOL to plasma core through separatrix on the structure of radial electric field is connected with plasma density reduction.

4) The radial electric field in edge plasma of small size divertor tokamak is plasma density dependence and the maximum radial electric field shear is found at low plasma density near separatrix.

5) The spikes in the radial electric field exist at the equatorial mid-plane in SN (Single Null) small size divertor tokamak. The existence of the spike is very important for $\mathrm{L}-\mathrm{H}$ transition and turbulent suppression in single null small size divertor tokamak plasma edge.

6) In the "NBI" discharge the toroidal (parallel) velocity at low plasma density is co-current (upward) direction. The absolute value of toroidal rotation is larger for lower edge plasma density. The reason for the deviation of toroidal velocity at low plasma density from the toroidal velocity at high plasma density can be explaining according as follow: the difference in toroidal velocities at different plasma densities refer to unbalance toroidal (parallel) particle flux caused by radial flux from the plasma core goes to SOL and divertor plates. This result is interesting since they might help explain, The shear of toroidal velocity at low plasma density near separatrix is smaller in case of the high plasma density which explains the larger L-H transition than for high plasma density.

7) The parallel (toroidal) rotation in edge plasma of small size divertor tokamak is plasma density dependence.

8) The simulation results shows the nature of the QH-regime of small size divertor tokamak which allows us to obtain high enough radial electric field shear for turbulence suppression and strong ITB formation. The radial electric field well in $\mathrm{QH}$-regime is so deep about $\sim 100 \mathrm{k} \mathrm{v} / \mathrm{m}$ and so narrow about $(1 \mathrm{~cm})$ compared with 20 - $40 \mathrm{k} \mathrm{v} / \mathrm{m}$ in H-regime.

The simulation of small size divertor tokamak plasma edge in QH-regime by using the B2SOLPS0.5.2D fluid transport code is the subject of the future work.

\section{REFERENCES}

[1] Tala, T.J., Heikkinen, J.A., Parail, V.V., Baranov, Yu.F. and Karttunen S.J. (2001) ITB formation in terms of $\omega_{\mathrm{E} \times \mathrm{B}}$ flow shear and magnetic shear $s$ on JET. Plasma Physics and Controlled Fusion, 43, 507-523. doi:10.1088/0741-3335/43/4/309

[2] Chankin, A.V., Coster, D.P., Dux, R., Fuchs, Ch., Haas, G., Herrmann, A., Horton, L.D., Kallenb and Schneider, W (2006) SOLPS modelling of ASDEX upgrade H-mode plasma. Plasma Physics and Controlled Fusion, 48, 839869. doi:10.1088/0741-3335/48/6/010

[3] Tala, T.J., Parail, V.V., Becoulet, A. Corrigan, G., Heading, D.J. and Contributors to the EFDA-JET Workprogramme (2002) Comparison of theory-based and semi-empirical transport modelling in JET plasmas with ITBs. Plasma Physics and Controlled Fusion, 44, A495-A500. doi:10.1088/0741-3335/44/5A/355

[4] Baranov, Yu.F., Garbet, X., Hawkes, N.C., Alper, B., Barnsley, R. and the JET EFDA Contributors (2004) On the link between the $q$-profile and internal transport barriers. Plasma Physics and Controlled Fusion, 46, 11811196. doi:10.1088/0741-3335/46/8/002

[5] Parail, V.V., Baranov, Yu.F., Challis, C.D., Cottrell, G.A., Fischer, B. and Ward D.J, (1999) Predictive modelling of JET optimized shear discharges. Nuclear Fusion, 39, 429437. doi:10.1088/0029-5515/39/3/310

[6] Crombé, K., Andrew, Y., Brix, M., Giroud, C., Hacquin, S. and Zastrow, K.D. (2005) Poloidal rotation dynamics, radial electric field, and neoclassical theory in the jet internal-transport-barrier region. Physical Review Letters, 95, 155003. doi:10.1103/PhysRevLett.95.155003

[7] Challis, C.D., Baranov, Yu.F., Conway, G.D., Gormezano, C., Gowers, C.W. and Zastrow, K.-D. (2001) Effect of $q$-profile modification by LHCD on internal transport barriers in JET. Plasma Physics and Controlled Fusion, 43, 861-879. doi:10.1088/0741-3335/43/7/303

[8] Challis, C.D., Litaudon, X., Tresset, G., Baranov, Yu.F., Bécoulet, A. and Contributors to the EFDA-JET Workprogramme (2002) Influence of the $q$-profile shape on plasma performance in JET. Plasma Physics and Controlled Fusion, 44, 1031-1055. doi:10.1088/0741-3335/44/7/301

[9] Hawkes, N.C., Andrew, Y., Challis, C.D., DeAngelis, R., Drozdov, V. and Contributors to the EFDA-JET Workprogramme (2002) The formation and evolution of extreme shear reversal in JET and its influence on local thermal transport. Plasma Physics and Controlled Fusion, 44, 1105,. doi:10.1088/0741-3335/44/7/304

[10] Burrell, K. et al. (1997) Effects of $\mathrm{E} \times \mathrm{B}$ velocity shear and magnetic shear on turbulence and transport in magnetic confinement devices. Physics of Plasmas, 4, 14991519. doi:10.1063/1.872367

[11] Connor, J.W., Fukuda, T., Garbet, X., Gormezano, C., Mukhovatov, V. and the ITB Database Group, the ITPA Topical Group on Transport and Internal Barrier Physics (2004) A review of internal transport barrier physics for steady-state operation of tokamaks. Nuclear Fusion, 44, R1-R49. doi:10.1088/0029-5515/44/4/R01

[12] Wolf, R. (2003) Internal transport barriers in tokamak plasmas. Plasma Physics and Controlled Fusion, 45, R1R91. doi:10.1088/0741-3335/45/1/201 
[13] Sakamoto, Y., Suzuki, T., Ide, S., Koide, Y., Takenaga, H. and Rewoldt, G. (2004) Properties of internal transport barrier formation in JT-60U. Nuclear Fusion, 44, 876-882. doi:10.1088/0029-5515/44/8/006

[14] Fujita, T., Ide, S., Kamada, Y., Suzuki, T., Oikawa, T., Takeji, S. and Fukuda, T. (2001) Quasisteady high-confinement reversed shear plasma with large bootstrap current fraction under full noninductive current drive condition in JT-60U. Physical Review Letters, 87, 085001. doi:10.1103/PhysRevLett.87.085001

[15] Bekheit, A.H. (2010) Simulation of radial electric field and internal transport barrier formation in small size divertor tokamak plasma edge. Journal of Fusion Energy, 29, 285-289. doi:10.1007/s10894-010-9274-2

[16] Bekheit, A.H. (2008) Simulation of small size divertor tokamak plasma edge including self-consistent electric fields. Journal of Fusion Energy, 27, 338-345. doi:10.1007/s10894-008-9148-z

[17] Rozhansky, V., Kaveeva, E., Voskoboynikov, S., Coster, D.P. and Schneider, R. (2001) Simulation of tokamak edge plasma including self-consistent electric fields. $\mathrm{Nu}$ clear Fusion, 41, 387. doi:10.1088/0029-5515/41/4/305

[18] Quigley, E.D., Peeters, A.G., Mc, P.J., Apostoliceanu, M., Hobirk, J. and the ASDEX Upgrade Team (2004) Formation criteria and positioning of internal transport barriers in ASDEX Upgrade. Nuclear Fusion, 44, 1189-1196. doi:10.1088/0029-5515/44/11/004

[19] Ernst, D.R., Bonoli, P.T. , Catto, P.J., Dorland, W., Fiore, C.L., Granetz, R.S. and Alcator C-Mod Group (2004) Role of trapped electron mode turbulence in internal transport barrier control in the Alcator C-Mod Tokamak. Physics of Plasmas, 11, 2637-26478. doi:10.1063/1.1705653

[20] Angioni, C., Peeters, A.G., Garbet, X., Manini, A., Ryter F and ASDEX Upgrade Team (2004) Density response to central electron heating: Theoretical investigations and experimental observations in ASDEX Upgrade. Nuclear Fusion, 44, 827-845. doi:10.1088/0029-5515/44/8/003

[21] Rozhansky, V., Kaveeva, E., Voskoboynikov, S., Counsell, G., Kirk, A. and the ASDEX Upgrade Team (2006) Modelling of radial electric field profile for different divertor configurations. Plasma Physics and Controlled Fusion, 48, 1425-1435. doi:10.1088/0741-3335/48/9/011

[22] Hirshman, S.P. and Sigmar, D.J. (1981) Neoclassical transport of impurities in tokamak plasmas. Nuclear $\mathrm{Fu}$ sion, 21, 1079-1210. doi:10.1088/0029-5515/21/9/003

[23] Burrell, K.H, West, W.P, Doyle, E.J, Austin, M.E, Gohil, P. and Zeng, L. (2004) Edge radial electric field structure in quiescent $\mathrm{H}$-mode plasmas in the DIII-D tokamak. Plasma Physics and Controlled Fusion, 46, A165-A178. 